https://doi.org/10.15407/ujpe66.7.551

T. ABEBE,$^{1}$ CH. GASHU ${ }^{2}$

${ }^{1}$ Department of Physics, Adama Science and Technology University

(P. O. Box 1888, Adama,Ethiopia; e-mail: tam1704@gmail.com)

2 Department of Physics, Jimma University

(P. O. Box 378, Jimma, Ethiopia)

\title{
GENERATION OF ENTANGLED LIGHT FROM A NONDEGENERATE THREE-LEVEL LASER COUPLED TO A TWO-MODE VACUUM RESERVOIR
}

\begin{abstract}
The quantum properties of a nondegenerate three-level cascade laser coupled to a two-mode vacuum reservoir are throughly analyzed with the use of the pertinent master equation and stochastic differential equations associated with the normal ordering. Particularly, the enhancement of squeezing and the amplification of photon entanglement of the two-mode cavity light are investigated. It is found that the two cavity modes are strongly entangled, and the degree of entanglement is directly related to the two-mode squeezing. Moreover, the squeezing and entanglement of the cavity radiation enhance with the rate of atomic injection.

Ke ywords: atomic coherence, quadrature squeezing, entanglement, mean photon number.
\end{abstract}

\section{Introduction}

Three-level lasers have been an interesting area of research over the years in light of its capability to produce radiations with various quantum properties $[1-$ 15]. The quantum properties of light are attributed to the atomic coherence that can be induced either by preparing the atoms initially in a coherent superposition of the top and bottom levels $[1,14,15]$, by coupling these levels of the atom with a strong classical pumping radiation [2,8-11], or by using these mechanisms together [5]. Such mechanism imposes a constraint on the populations of atoms in the bottom and top levels in which transitions to and from could not be made in the electric dipole approximation. The classical pumping radiation, therefore, contributes to the observed nonclassical properties by facilitating the atomic population transfer pathway in which the induced correlation is transferred to the emitted photons.

(C) T. ABEBE, CH. GASHU, 2021

ISSN 2071-0194. Ukr. J. Phys. 2021. Vol. 66, No. 7
Moreover, the quantum entanglement has been considered as the nonlocality aspect of quantum correlations with no classical similarity. This wonderful feature was investigated in the seminal paper of Einstein-Podolsky-Rosen (EPR) [16]. After that, Bell recognized that the entanglement leads to experimentally testable deviations of quantum mechanics from classical physics [17]. As we know, the generation of the entanglement has recently attracted great interest, since it plays a key role in the quantum information processing [18-22]. Particularly, much attention has been paid to the generation of a continuousvariable entanglement, as it might be easier manipulated than the discrete counterparts, quantum bits, in order to perform the quantum information processing. Hence, with the advent of quantum information theory, the entanglement was known as a resource for many applications such as quantum cryptography [23], quantum computation and communication [24], quantum dense coding [25], quantum teleportation [26], entanglement swapping [27], sen- 


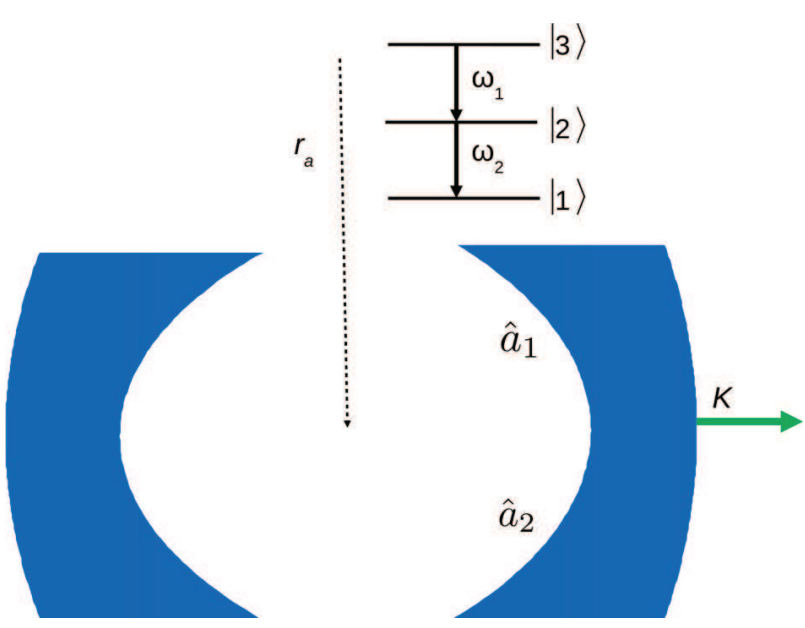

Fig. 1. Schematic representation of a nondegenerate threelevel laser coupled to a two-mode vacuum reservoir. Here, $\hat{a}_{1}\left(\hat{a}_{2}\right)$ is the annihilation operator for one of the modes of the cavity radiation, $r_{a}$ represents the rate at which the atoms are injected into the cavity, and $\kappa$ is the cavity damping constant taken to be the same for both transitions. Moreover, $|3\rangle,|2\rangle$, and $|1\rangle$, represent the top, intermediate, and bottom levels of a three-level atom in a cascade configuration, respectively. We consider the case for which the two cavity-mode are at resonance with the two transitions $|3\rangle \rightarrow|2\rangle$ and $|2\rangle \rightarrow|1\rangle$ having transition frequencies $\omega_{1}$ and $\omega_{2}$. The two modes $a_{1}$ and $a_{2}$ to be at resonance with the two transitions $|3\rangle \rightarrow|2\rangle$ and $|2\rangle \rightarrow|1\rangle$ are dipole-allowed, and the direct transition between level $|3\rangle$ and level $|1\rangle$ to be dipole-forbidden

sitive measurements [28], and quantum telecloning [29]. Hence, an interest in understanding the entanglement creation and quantification has gained the attention of several authors[10,13-15,30-35]. In general, the degree of entanglement degrades, as it interacts with the environment. On the other hand, the efficiency of the quantum information processing highly depends on the degree of entanglement. As a consequence, it is desirable to generate strongly entangled continuous-variable states which can survive from environmental noise.

Moreover, many schemes have been proposed to produce a strong entangled light from a three-level laser using different techniques theoretically $[6,7,13-$ $15,35]$. Some authors have studied the effect of a parametric amplifier on the quantum properties of light generated by a three-level laser [7]. Alebachew has found that the parametric amplifier in the laser cavity increases the degree of entanglement [7]. This work has been confined to the case in which the steadystate analysis is above the threshold condition. Howe- ver, the solutions for the cavity-mode variables cannot be above the threshold condition of the steadystate analysis $[9,16,18]$.

In this paper, we consider a nondegenerate threelevel laser coupled to a vacuum reservoir with an injected coherence system. Here, we carry out our analysis by applying the pertinent master equation describing the dynamics of the optical device [36]. Employing this master equation, we obtain the stochastic differential equations. Hence, using the solutions of these equations, we determined the correlation property of noise forces associated with normal ordering. Finally, with the help of the steady-state solutions of the $c$-number cavity mode operators, we studied the quadrature squeezing and entanglement using logarithmic negativity criteria.

\section{Hamiltonian and Master Equation}

The interaction of a nondegenerate three-level atom with two-mode cavity radiation can be expressed in the interaction picture with the rotating-wave approximation (RWA) with the Hamiltonian of the form

$\hat{H}_{I}=i g\left[|3\rangle\left\langle 2\left|\hat{a}_{1}-\hat{a}_{1}^{\dagger}\right| 2\right\rangle\langle 3|+| 2\rangle\left\langle 1\left|\hat{a}_{2}-\hat{a}_{2}^{\dagger}\right| 1\right\rangle\langle 2|\right]$,

where $g$ is a coupling constant which is taken to be the same for both transitions, whereas $\hat{a}_{1}\left(\hat{a}_{1}^{\dagger}\right)$ and $\hat{a}_{2}\left(\hat{a}_{2}^{\dagger}\right)$ are the annihilation (creation) operators of the corresponding cavity modes. In writing Eq. (1), we have considered $\hbar=1$ for the sake of simplicity only.

In this paper, we take the initial state of a threelevel atom to be $\left|\psi_{A}(0)\right\rangle=C_{3}(0)|3\rangle+C_{1}(0)|1\rangle$. Hence, the initial density operator for a single atom has the form

$\hat{\rho}_{A}(0)=\rho_{33}^{(0)}|3\rangle\left\langle 3\left|+\rho_{31}^{(0)}\right| 3\right\rangle\left\langle 1\left|+\rho_{13}^{(0)}\right| 1\right\rangle\left\langle 3\left|+\rho_{11}^{(0)}\right| 1\right\rangle\langle 1|$,

where $\rho_{33}^{(0)}=\left|C_{3}\right|^{2}$ and $\rho_{11}^{(0)}=\left|C_{1}\right|^{2}$ are, respectively, the probabilities for the atom to be initially in the upper or lower levels, $\rho_{31}^{(0)}=C_{3} C_{1}^{*}$, and $\rho_{13}^{(0)}=C_{1} C_{3}^{*}$. Actually, this assumption corresponds to a situation in which the three-level atom is initially prepared in a coherent superposition of the top and bottom levels.

In addition, we will consider the case where such atoms are injected into a cavity at the constant rate $r_{a}$ and removed after sometime $\tau$, which is long enough for the atoms to decay spontaneously to levels other than the middle or lower level. The

ISSN 2071-0194. Ukr. J. Phys. 2021. Vol. 66, No. 7 
spontaneous decay rate $\gamma$ is taken to be the same for the two upper levels. In the good cavity limit, $\gamma \gg \kappa$, where $\kappa$ is the cavity damping constant, the cavity mode variables change slowly compared with the atomic variables. Hence the atomic variables will reach the steady state in a relatively short time. The time derivative of such variables can then be set to zero, while keeping the remaining terms at the time $t$. This procedure is referred as the adiabatic approximation scheme. Since the coupling constant is taken to be small, we restrict ourselves to a linear analysis that includes dropping the higher order terms in $g$. Employing the linear and adiabatic approximation schemes in the good cavity limit, we get the equation of evolution of the density operator for the cavity modes in the absence of damping through the coupled mirror in the form [14]

$$
\begin{aligned}
& \dot{\hat{\rho}}_{1}(t)=\frac{A \rho_{33}^{(0)}}{2}\left[2 \hat{a}_{1}^{\dagger} \hat{\rho} \hat{a}_{1}-\hat{a}_{1} \hat{a}_{1}^{\dagger} \hat{\rho}-\hat{\rho} \hat{a}_{1} \hat{a}_{1}^{\dagger}\right]+ \\
& +\frac{A \rho_{11}^{(0)}}{2}\left[2 \hat{a}_{2} \hat{\rho} \hat{a}_{2}^{\dagger}-\hat{a}_{2}^{\dagger} \hat{a}_{2} \hat{\rho}-\hat{\rho} \hat{a}_{2}^{\dagger} \hat{a}_{2}\right]- \\
& -\frac{A \rho_{31}^{(0)}}{2}\left[2 \hat{a}_{2} \hat{\rho} \hat{a}_{1}-\hat{\rho} \hat{a}_{1} \hat{a}_{2}-\hat{a}_{1} \hat{a}_{2} \hat{\rho}\right]- \\
& -\frac{A \rho_{13}^{(0)}}{2}\left[2 \hat{a}_{1}^{\dagger} \hat{\rho} \hat{a}_{2}^{\dagger}-\hat{\rho} \hat{a}_{1}^{\dagger} \hat{a}_{2}^{\dagger}-\hat{a}_{1}^{\dagger} \hat{a}_{2}^{\dagger} \hat{\rho}\right]
\end{aligned}
$$

where $A=\frac{2 g^{2} r_{a}}{\gamma^{2}}$ is the linear gain coefficient, and, for convenience, we have set $\rho_{31}^{(0)}=\rho_{31}^{(0) *}$.

Next, we consider a system coupled with a twomode vacuum reservoir. The density operator which is extracted from the vacuum reservoir by the partial trace operation is [37]

$$
\begin{aligned}
& \dot{\hat{\rho}}_{2}(t)=\frac{\kappa}{2}\left[2 \hat{a}_{1} \hat{\rho} \hat{a}_{1}^{\dagger}-\hat{a}_{1}^{\dagger} \hat{a}_{1} \hat{\rho}-\hat{\rho} \hat{a}_{1}^{\dagger} \hat{a}_{1}\right]+ \\
& +\frac{\kappa}{2}\left[2 \hat{a}_{2} \hat{\rho} \hat{a}_{2}^{\dagger}-\hat{a}_{2}^{\dagger} \hat{a}_{2} \hat{\rho}-\hat{\rho} \hat{a}_{2}^{\dagger} \hat{a}_{2}\right] .
\end{aligned}
$$

Finally, using Eqs. (3), and (4), the master equation for the system takes the form:

$$
\begin{aligned}
& \dot{\hat{\rho}}(t)=\frac{\kappa}{2}\left[2 \hat{a}_{1} \hat{\rho} \hat{a}_{1}^{\dagger}-\hat{a}_{1}^{\dagger} \hat{a}_{1} \hat{\rho}-\hat{\rho} \hat{a}_{1}^{\dagger} \hat{a}_{1}\right]+ \\
& +\frac{1}{2} A \rho_{33}^{(0)}\left[2 \hat{a}_{1}^{\dagger} \hat{\rho} \hat{a}_{1}-\hat{a}_{1} \hat{a}_{1}^{\dagger} \hat{\rho}-\hat{\rho} \hat{a}_{1} \hat{a}_{1}^{\dagger}\right]+ \\
& +\frac{1}{2}\left(A \rho_{11}^{(0)}+\kappa\right)\left[2 \hat{a}_{2} \hat{\rho} \hat{a}_{2}^{\dagger}-\hat{a}_{2}^{\dagger} \hat{a}_{2} \hat{\rho}-\hat{\rho} \hat{a}_{2}^{\dagger} \hat{a}_{2}\right]+ \\
& +\frac{A \rho_{31}^{(0)}}{2}\left[2 \hat{a}_{2} \hat{\rho} \hat{a}_{1}-\hat{\rho} \hat{a}_{1} \hat{a}_{2}-\hat{a}_{1} \hat{a}_{2} \hat{\rho}\right]+
\end{aligned}
$$$$
\text { ISSN 2071-0194. Ukr. J. Phys. 2021. Vol. 66, No. } 7
$$

$+\frac{A \rho_{13}^{(0)}}{2}\left[2 \hat{a}_{1}^{\dagger} \hat{\rho} \hat{a}_{2}^{\dagger}-\hat{\rho} \hat{a}_{1}^{\dagger} \hat{a}_{2}^{\dagger}-\hat{a}_{1}^{\dagger} \hat{a}_{2}^{\dagger} \hat{\rho}\right]$

The above master equation can be used to derive the time variation for the expectation values of various system operators. The terms proportional to $\rho_{33}^{(0)}$ and $\rho_{11}^{(0)}$ describe the gain of cavity light for mode $a_{1}$ and the loss for mode $a_{2}$, respectively. The terms proportional to $\rho_{31}^{(0)}$ are related to the correlation of the generated radiation that indicates the existence of quantum features. These terms are responsible for the squeezing obtained in the cascade laser system. Furthermore, the terms proportional to $\kappa$ describe the damping of cavity modes due to their coupling with a two-mode vacuum reservoir via a single-port mirror.

It proves to be useful to introduce a new parameter which relates the probabilities of the atom to be in the upper and lower levels. We define the parameter $\eta$ such that $\rho_{33}^{(0)}=\frac{1-\eta}{2}$ with $-1<\eta<1$. For three-level atoms initially in a coherent superposition of the top and bottom levels, one obtains: $\rho_{11}^{(0)}=\frac{1+\eta}{2}$. Then, in view of the relation $\left|\rho_{31}^{(0)}\right|^{2}=\rho_{33}^{(0)} \rho_{11}^{(0)}$, one easily finds $\rho_{31}^{(0)}=\frac{1}{2} \sqrt{1-\eta^{2}}$.

Employing the master equation (5), the evolution of the two-mode cavity radiation in terms of $c$-number variables associated with the normal ordering, $\alpha_{1}(t)$ and $\alpha_{2}(t)$ can be expressed in the form

$\frac{d}{d t} \alpha_{1}(t)=-\Gamma_{+} \alpha_{1}(t)-\xi_{+} \alpha_{2}^{*}(t)+f_{1}(t)$,
$\frac{d}{d t} \alpha_{2}(t)=-\Gamma_{-} \alpha_{2}(t)-\xi_{-} \alpha_{1}^{*}(t)+f_{2}(t)$,

where

$\Gamma_{ \pm}=\frac{\kappa}{2}-\frac{A}{4}(\eta \pm 1)$

$\xi_{ \pm}= \pm \frac{A}{4} \sqrt{1-\eta^{2}}$

$f_{1}(t)$ and $f_{2}^{*}(t)$ are noise forces whose properties remain to be determined, $\alpha_{1}(t)$ and $\alpha_{2}(t)$ are the $c$ number variables corresponding to the cavity-mode operators $\hat{a}_{1}$ and $\hat{a}_{2}$.

Following the procedure described in Ref. [13], we obtain:

$\alpha_{1}(t)=A_{+}(t) \alpha_{1}(0)+B_{+}(t) \alpha_{2}^{*}(0)+F_{+}(t)+W_{+}(t)$,

$\alpha_{2}(t)=A_{-}(t) \alpha_{2}(0)+B_{-}(t) \alpha_{1}^{*}(0)+F_{-}(t)+W_{-}(t)$, 
where

$$
\begin{aligned}
& A_{ \pm}(t)=\frac{1}{2}\left[(1 \pm p) e^{-\lambda_{-} t}+(1 \mp p) e^{-\lambda_{+} t}\right] \\
& B_{ \pm}(t)=\frac{q_{ \pm}}{2}\left[e^{-\lambda_{+} t}-e^{-\lambda_{-} t}\right] \\
& F_{+}(t)=\frac{1}{2} \int_{0}^{t}\left[(1+p) e^{-\lambda_{-}\left(t-t^{\prime}\right)}+\right. \\
& \left.+(1-p) e^{-\lambda_{+}\left(t-t^{\prime}\right)}\right] f_{1}\left(t^{\prime}\right) d t^{\prime} \\
& F_{-}(t)=\frac{1}{2} \int_{0}^{t}\left[(1-p) e^{-\lambda_{-}\left(t-t^{\prime}\right)}+\right. \\
& \left.+(1+p) e^{-\lambda_{+}\left(t-t^{\prime}\right)}\right] f_{2}\left(t^{\prime}\right) d t^{\prime}, \\
& W_{+}(t)=\frac{q_{+}}{2} \int_{0}^{t}\left[e^{-\lambda_{+}\left(t-t^{\prime}\right)}-e^{-\lambda_{-}\left(t-t^{\prime}\right)}\right] f_{2}^{*}\left(t^{\prime}\right) d t^{\prime}, \\
& W_{-}(t)=\frac{q_{-}}{2} \int_{0}^{t}\left[e^{-\lambda_{+}\left(t-t^{\prime}\right)}-e^{-\lambda_{-}\left(t-t^{\prime}\right)}\right] f_{1}^{*}\left(t^{\prime}\right) d t^{\prime},
\end{aligned}
$$

with $p=1 / \eta, q_{ \pm}= \pm \sqrt{1-\eta^{2}} / \eta, \lambda_{ \pm}=\frac{\kappa}{2}+\frac{A}{4}(\eta \pm 1)$. The correlation properties of the noise forces $f_{1}(t)$ and $f_{2}(t)$ associated with the normal ordering satisfy the relations

$$
\begin{aligned}
& \left\langle f_{1}(t)\right\rangle=\left\langle f_{2}(t)\right\rangle=\left\langle f_{1}\left(t^{\prime}\right) f_{1}(t)\right\rangle=0, \\
& \left\langle f_{2}(t) f_{2}\left(t^{\prime}\right)\right\rangle=\left\langle f_{1}^{*}(t) f_{2}\left(t^{\prime}\right)\right\rangle=0, \\
& \left\langle f_{2}\left(t^{\prime}\right) f_{2}^{*}(t)\right\rangle=\left\langle f_{2}^{*}\left(t^{\prime}\right) f_{1}(t)\right\rangle=0, \\
& \left\langle f_{1}\left(t^{\prime}\right) f_{1}^{*}(t)\right\rangle=\frac{A}{2}(1-\eta) \delta\left(t-t^{\prime}\right), \\
& \left\langle f_{2}\left(t^{\prime}\right) f_{1}(t)\right\rangle=-\frac{1}{2} \xi_{-} \delta\left(t-t^{\prime}\right) .
\end{aligned}
$$

\section{Quadrature Fluctuations}

In this section, following a similar approach, the evolution of the two-mode quadrature squeezing is evaluated, and the comparison with the steady state case is made whenever possible.

Generally, a two-mode cavity radiation can be described by the operator

$\hat{c}=\frac{1}{\sqrt{2}}\left(\hat{a}_{1}+\hat{a}_{2}\right)$.

where $\hat{a}_{1}$ and $\hat{a}_{2}$ represent the separate modes. With this consideration, the squeezing properties of the cavity radiation can be studied applying the quadrature operators defined by

$\hat{c}_{+}=\left(\hat{c}^{\dagger}+\hat{c}\right)$,

$\hat{c}_{-}=i\left(\hat{c}^{\dagger}-\hat{c}\right)$.

These quadrature operators satisfy the commutation relation $\left[\hat{c}_{+}, \hat{c}_{+}=2 i\right]$. On the basis of these definitions, a two-mode light is said to be in a two-mode vacuum state, if either $\Delta c_{+}^{2}<1$ or $\Delta c_{-}^{2}<1$. Taking the cavity modes to be initially in a two-mode vacuum state, the variances of the quadrature operators (24) and (25) can be expressed in terms of the $c$-number variables associated with the normal ordering as

$\Delta c_{ \pm}^{2}=1+\left\langle\alpha_{1}^{*}(t) \alpha_{1}(t)\right\rangle+\left\langle\alpha_{2}^{*}(t) \alpha_{2}(t)\right\rangle+\left\langle\alpha_{1}^{*}(t) \alpha_{2}(t)\right\rangle+$ $+\left\langle\alpha_{1}(t) \alpha_{2}^{*}(t)\right\rangle \pm\left\{\left\langle\alpha_{1}(t) \alpha_{2}(t)\right\rangle+\left\langle\alpha_{1}^{*}(t) \alpha_{2}^{*}(t)\right\rangle+\right.$

$\left.+\frac{1}{2}\left[\left\langle\alpha_{1}^{2}(t)\right\rangle+\left\langle\alpha_{2}^{2}(t)\right\rangle+\left\langle\alpha_{1}^{* 2}(t)\right\rangle+\left\langle\alpha_{2}^{* 2}(t)\right\rangle\right]\right\}$.

It is necessary to determine the various correlations described in Eq. (26) by using Eqs. (10) and (11). In line with this, assuming that the cavity is initially in a two-mode vacuum state, and the noise force at time $t$ is not statistically related to the cavity mode variables at earlier times, one can readily verify that

$\left\langle\alpha_{1}^{2}\right\rangle=\left\langle\alpha_{2}^{2}\right\rangle=\left\langle\alpha_{1} \alpha_{2}^{*}\right\rangle=\left\langle\alpha_{1}^{*} \alpha_{2}\right\rangle=0$,

$\left\langle\alpha_{1}^{*} \alpha_{1}\right\rangle=\frac{A(1-\eta)^{2}}{\kappa(\kappa+A \eta)}+\frac{A\left(1-\eta^{2}\right)}{2 \kappa(2 \kappa+A \eta)}$,

$\left\langle\alpha_{2}^{*} \alpha_{2}\right\rangle=\frac{A\left(1-\eta^{2}\right)}{2 \kappa(2 \kappa+A \eta)}-\frac{A\left(1-\eta^{2}\right)}{4 \kappa(\kappa+A \eta)}$,

$\left\langle\alpha_{1} \alpha_{2}\right\rangle=\frac{A \sqrt{1-\eta^{2}}}{2 \kappa(2 \kappa+A \eta)}-\frac{A(1-\eta) \sqrt{\left(1-\eta^{2}\right)}}{4 \kappa(\kappa+A \eta)}$

The results in Eq. (27), namely, $\left\langle\alpha_{1}^{2}\right\rangle=\left\langle\alpha_{2}^{2}\right\rangle=0$, represent the intercorrelation of each mode. It is a well-known fact that the atoms in the quantum system interact through the exchange of photons. Here, a correlation among similar states of the cavity radiation vanishes, which, in other words, means the absence of intercorrelation interactions among the atoms, as they are assumed to leave the cavity within a short time interval. The results in Eqs. (28) and (29) indicate the steady-state mean photon number of the cavity modes $\hat{a}_{1}$ and $\hat{a}_{2}$, respectively. Moreover, Eq. (30) indicates the correlation between the two cavity modes. The nonclassical features of the

ISSN 2071-0194. Ukr. J. Phys. 2021. Vol. 66, No. 7 
cavity radiation are attributed to the correlation between the two cavity modes given in this equation.

Furthermore, with the help of Eq. (27), one can easily obtain

$$
\begin{aligned}
& \Delta c_{ \pm}^{2}=1+\left\langle\alpha_{1}^{*}(t) \alpha_{1}(t)\right\rangle+\left\langle\alpha_{2}^{*}(t) \alpha_{2}(t)\right\rangle \pm \\
& \pm 2\left\langle\alpha_{1}(t) \alpha_{2}(t)\right\rangle .
\end{aligned}
$$

We clearly see from Fig. 2 that the squeezing occurs for values of $\eta$ between 0 and 1 . This corresponds to the case where the atoms are initially prepared in such a way that there are more atoms in the bottom level than in the upper level. We also note that a significant squeezing exists, when almost a half of the atoms are initially on the upper level. Hence, we observe that a light produced by a nondegenerate three-level laser can exhibit a substantial degree of squeezing. This indicates that the more the atoms are injected into the cavity at a time, the more the degree of squeezing of the cavity radiation would be. The maximum squeezing occurs when the atoms are prepared with the initial coherence very close to the maximum possible value in this case. Moreover, it is possible to realize that the degree of squeezing increases with the linear gain coefficient. In particular, the maximum $72-\%$ squeezing occurs at $\eta=0.1$, when $A=1000$ and $\kappa=0.8$.

On the basis of the definition of the parameter $\eta$, we note that, for $\eta=0, \rho_{33}^{(0)}=\rho_{11}^{(0)}=\rho_{13}^{(0)}=0.5$, which corresponds to the maximum initial atomic coherence. But, for $\eta=1, \rho_{33}^{(0)}=\rho_{13}^{(0)}=0$ and $\rho_{11}^{(0)}=1$, which indicates that there is no injected atomic coherence at the beginning. It is not difficult to see from Fig. 2 that no squeezing property is exhibited, when the atoms are initially prepared with maximum or minimum atomic coherence.

\section{Entanglement}

Here, we study the degree of entanglement of the twomode cavity light produced by a nondegenerate threelevel cascade laser whose cavity contains a parametric amplifier. A pair of particles is taken to be entangled in quantum theory, if its states cannot be expressed as a product of the states of its individual constituents. The preparation and manipulation of these entangled states that have nonclassical and nonlocal properties lead to a better understanding of the basic quantum principles [13, 14], if the density

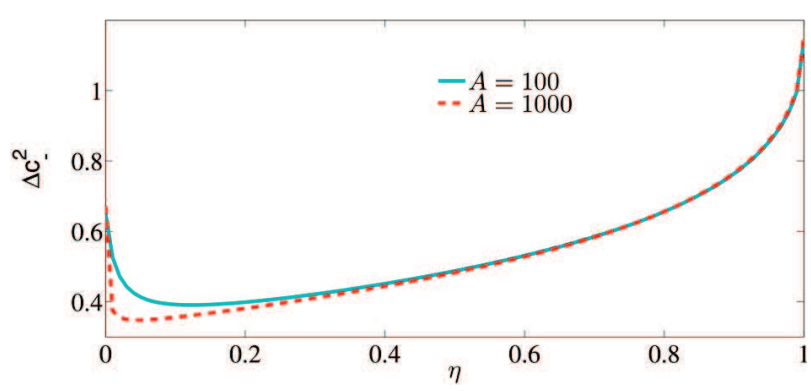

Fig. 2. (Color online) Plots of the minus quadrature variance of the cavity radiation in the steady state versus $\eta$ for $\kappa=0.8$

operator for the combined state cannot be described as the product of the density operators of the constituents,

$\hat{\rho} \neq \sum_{j} P_{j} \hat{\rho}_{j}^{(1)} \bigotimes \hat{\rho}_{j}^{(2)}$

in which $P_{j} \geq 0$, and $\sum_{j} P_{j}=1$ is set to ensure the normalization of the combined density of states.

A criterion to study the entanglement is the logarithmic negativity which is used for a two-mode continuous variables based on the negativity of the partial transposition $[35,38]$. The negative partial transpose must be parallel with respect to the entanglement monotone in order to obtain the degree of entanglement. The logarithmic negativity is combined with a negative partial transpose in another case where $V$ represents the smallest eigenvalue of the symplectic matrix [38]:

$V_{S}=\sqrt{\frac{\sigma-\sqrt{\left(\sigma^{2}-4 \operatorname{det} \Gamma\right)}}{2}}$,

where the invariant and covariance matrices are, respectively, denoted as:

$\sigma=\operatorname{det} \Omega_{1}+\operatorname{det} \Omega_{2}-2 \operatorname{det} \Omega_{12}$,

$\Gamma=\left(\begin{array}{cc}\Omega_{1} & \Omega_{12} \\ \Omega_{12}^{T} & \Omega_{2}\end{array}\right)$

in which $\Omega_{1}$ and $\Omega_{2}$ are the covariance matrices describing each mode separately, while $\Omega_{12}$ are the intermodal correlations. The elements of the matrix in equation (35) are given by:

$\Gamma_{i j}=1 / 2\left\langle\hat{X}_{i} \hat{X}_{j}+\hat{X}_{j} \hat{X}_{i}\right\rangle-\left\langle\hat{X}_{i}\right\rangle\left\langle\hat{X}_{j}\right\rangle$, 


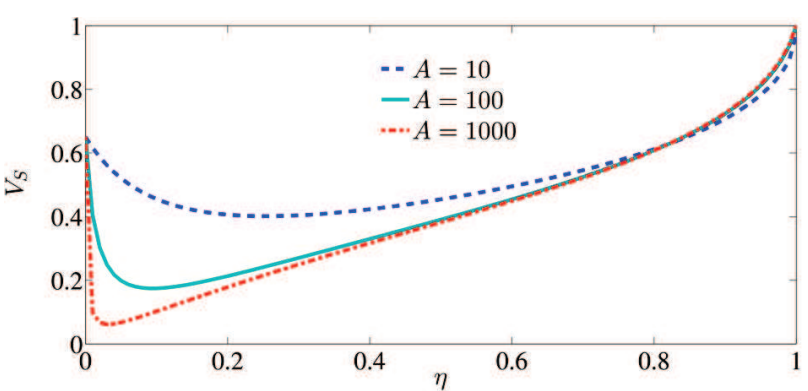

Fig. 3. (Color online) Plots of the smallest eigenvalue $V_{S}$ versus $\eta$ for $\kappa=0.8$ and for different values of the linear gain coefficient $A$

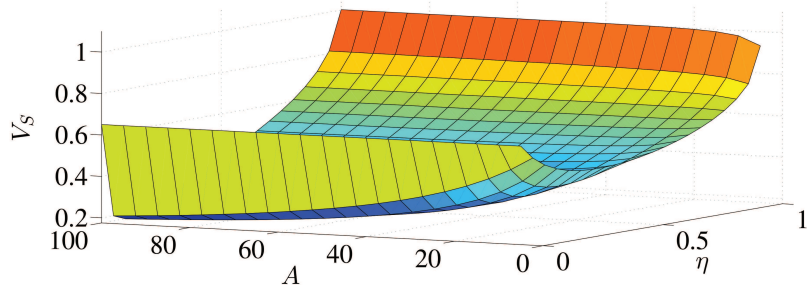

Fig. 4. (Color online) Plot of the smallest eigenvalue $V_{S}$ versus $\eta$ and the linear gain coefficient $A$ for $\kappa=0.8$

in which $i, j=1,2,3,4$. The quadrature operators are defined as

$\hat{X}_{1}=\hat{a}_{1}+\hat{a}_{1}^{\dagger}, \quad \hat{X}_{2}=i\left(\hat{a}_{1}^{\dagger}-\hat{a}_{1}\right)$,

$\hat{X}_{3}=\hat{a}_{2}+\hat{a}_{2}^{\dagger}, \quad \hat{X}_{4}=i\left(\hat{a}_{2}^{\dagger}-\hat{a}_{2}\right)$.

With this introduction, the extended covariance matrix becomes

$\Gamma=\left(\begin{array}{cccc}\Sigma_{1} & 0 & \Sigma_{12} & 0 \\ 0 & \Sigma_{1} & 0 & -\Sigma_{12} \\ \Sigma_{12} & 0 & \Sigma_{2} & 0 \\ 0 & -\Sigma_{12} & 0 & \Sigma_{2}\end{array}\right)$,

where $\Sigma_{1}=2\left\langle\alpha_{1}^{*} \alpha_{1}\right\rangle+1, \Sigma_{12}=2\left\langle\alpha_{1} \alpha_{2}\right\rangle, \Sigma_{2}=$ $=2\left\langle\alpha_{2}^{*} \alpha_{2}\right\rangle+1$ are $c$-number variables associated with the normal ordering. The logarithmic negativity is defined as:

$E_{N}=\max \left[0,-\log _{2} V_{S}\right]$.

The entanglement is achieved, when $E_{N}$ is positive within the region of the lowest eigenvalue of the covariance matrix $V_{S}<1[35,38]$.

In view of Eqs. (34) and (38), one can readily show that:

$\operatorname{det} \Omega_{1}=1+4\left\langle\alpha_{1}^{*}(t) \alpha_{1}(t)\right\rangle\left[\left\langle\alpha_{1}^{*}(t) \alpha_{1}(t)\right\rangle+1\right]$, $\operatorname{det} \Omega_{2}=1+4\left\langle\alpha_{2}^{*}(t) \alpha_{2}(t)\right\rangle\left[\left\langle\alpha_{2}^{*}(t) \alpha_{2}(t)\right\rangle+1\right]$,

$\operatorname{det} \Omega_{12}=-4\left\langle\alpha_{1}(t) \alpha_{2}(t)\right\rangle^{2}$.

It is also possible to establish that:

$\operatorname{det} \Gamma=\left[\sqrt{\operatorname{det} \Omega_{1} \operatorname{det} \Omega_{2}}-\sqrt{\operatorname{det} \Omega_{12}^{T} \operatorname{det} \Omega_{12}}\right]^{2}$.

As is seen in Fig. 3, the degree of entanglement increases for smaller values for the initial preparation of atoms, but decreases for larger values. It can also be seen that larger values of the linear gain coefficient produce a robust entangled light. The maximum achievable degree of entanglement of light in this case is $96 \%$, and this occurs for $A=1000$ and $\eta=0.02$. This criterion also predicts no entanglement for $\eta=1$, no matter how we manipulate the rate of atomic injection in the absence of a parametric amplifier (NLC) [14].

On the other hand, Fig. 4 shows that the effect of a parametric amplifier for large values of the linear gain coefficient does not produce considerable changes in the degree of entanglement. Hence, the maximum achievable degree of entanglement of light in this case is $82 \%$, and this occurs for $A=100$ and $\eta=0.13$. Here, the entanglement occurs at the minimum atomic coherence which represents the absence of photons in the cavity for very large values of the linear gain coefficient (rate of atomic injection).

\section{Photon Statistics}

Here, we study the statistical properties of the cavity radiation of a three-level cascade laser such as the mean photon number, Mandel's $Q$-factor, the inensity difference fluctuations, and the normalized secondorder correlation function for the system under consideration.

\subsection{Mean photon number}

In order to know about the brightness of the generated light, it is necessary to study the mean number of photon pairs describing the two-mode cavity radiation that can be defined as

$\bar{N}=\left\langle\hat{c}^{\dagger}(t) \hat{c}(t)\right\rangle$

It then follows that

$\bar{N}=\frac{1}{2}\left[\left\langle\alpha_{1}^{*}(t) \alpha_{1}(t)\right\rangle+\left\langle\alpha_{2}^{*}(t) \alpha_{2}(t)\right\rangle\right]$. 
Since $\left\langle\alpha_{1}^{*}(t) \alpha_{1}(t)\right\rangle$ and $\left\langle\alpha_{2}^{*}(t) \alpha_{2}(t)\right\rangle$ represent the mean photon numbers in mode $a_{1}$ and mode $a_{2}$, respectively, $\bar{N}$ can be interpreted as the mean number of photon pairs. As it can be seen from Eq. (43), the term that contains $\varepsilon$ represents the contribution from the external driving coherent light of a parametric amplifier to the total mean photon number. Therefore, it is easy to verify that Eq. (43) represents the mean number of photon pairs of the system.

It is not difficult to see from Fig. 5 that the mean number of photon pairs increases with the linear gain coefficient for $\kappa=0.8$ and for smaller values of $\eta$, but it decreases for larger values. Moreover, Fig. 6 clearly indicates that the mean photon number of the two-mode cavity light increases with the linear gain coefficient, $A$. It is quite interesting to note from this figure that the linear gain coefficient enhances the mean photon number over the laser system by a large number of mean photon numbers. Therefore, the increase in the mean photon number is observed in a region, where the degrees of two-mode squeezing and entanglement are significant making the system under consideration a viable source of intense squeezed, as well as entangled, light.

\subsection{Mandel's Q-factor}

It is a common experience that a nonclassical photon number correlation can be studied applying the measure of the departure of the photon statistics from the Poisson character. The measure of a departure can be represented by Mandel's $Q$-factor defined as

$Q=\frac{\left\langle(\Delta \hat{n})^{2}\right\rangle-\langle\hat{n}\rangle}{\langle\hat{n}\rangle}$,

where $\hat{n}=\hat{c}^{\dagger} \hat{c}$ is the photon number operator of a two-mode cavity radiation. It is not difficult to verify that Eq. (44) can be expressed by putting the operators in the normal ordering as

$Q=\frac{\left\langle\hat{c}^{\dagger} \hat{c}^{2}\right\rangle+\left\langle\hat{c}^{\dagger} \hat{c}\right\rangle^{2}}{\left\langle\hat{c}^{\dagger} \hat{c}\right\rangle}$

where $\hat{c}=\frac{1}{\sqrt{2}}\left(\hat{a}_{1}+\hat{a}_{2}\right)$ is the annihilation operator that describes the two-mode cavity radiation. In view of this, the normal ordering of the operators would not be altered, since $\hat{a}_{1}$ and $\hat{a}_{2}$ commute. Hence, it is possible to put the resulting expression in terms of $c$ -

ISSN 2071-0194. Ukr. J. Phys. 2021. Vol. 66, No. 7

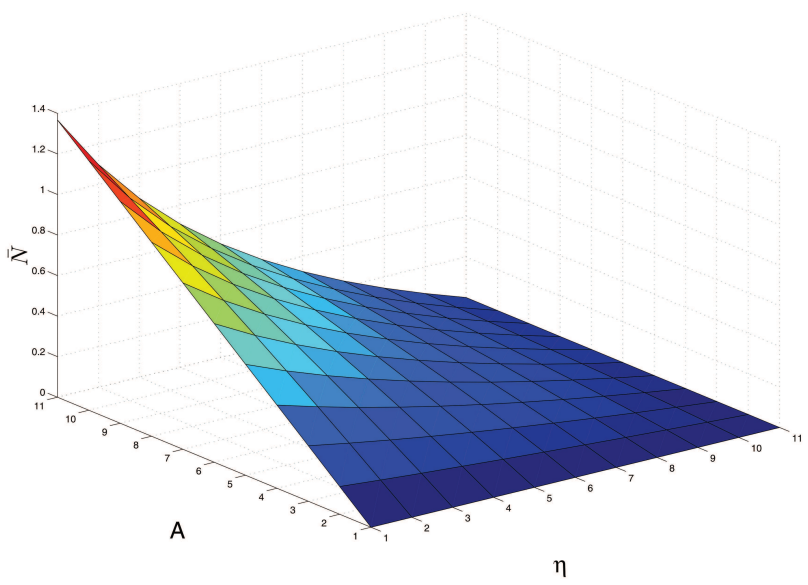

Fig. 5. (Color online) Plot of the mean photon number $N$ versus $\eta$ and the linear gain coefficient $A$ for $\kappa=0.8$

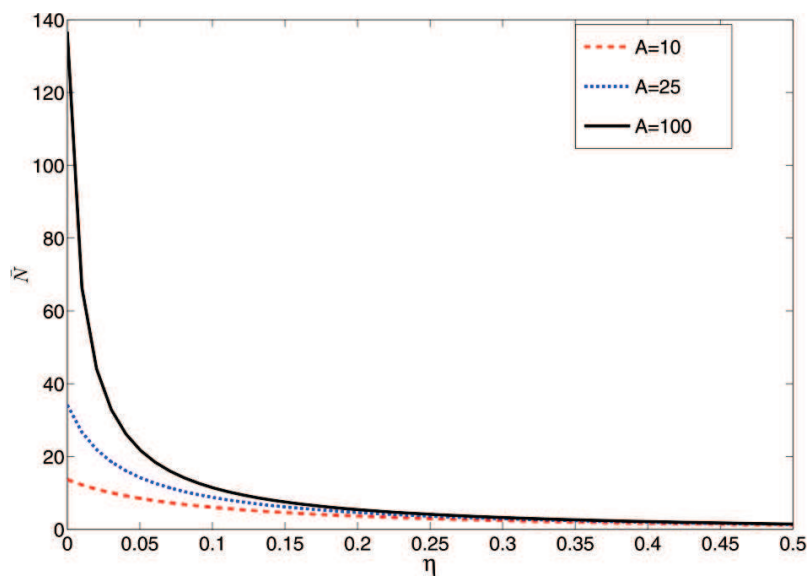

Fig. 6. (Color online) Plots of the mean photon number $N$ versus $\eta$ for $\kappa=0.8$ and for different values of the linear gain coefficient $A$

number variables associated with the normal order as

$Q=\frac{\left\langle\gamma^{* 2}(t) \gamma^{2}(t)\right\rangle+\left\langle\gamma^{*}(t) \gamma(t)\right\rangle^{2}}{\left\langle\gamma^{*}(t) \gamma(t)\right\rangle}$

where $\gamma=\frac{1}{\sqrt{2}}\left(\alpha_{1}(t)+\alpha_{2}(t)\right)$. Hence, employing Eqs. (10) and (11), one obtains

$Q=\bar{N}+\frac{\left\langle\alpha_{1}(t) \alpha_{2}(t)\right\rangle^{2}}{\bar{N}}$.

It is well known that the negativity of Mandel's parameter refers to a sub-Poissonian character of the photon statistics that essentially refers to a nonclassical property. Since the mean number of photon pairs and $\left\langle\alpha_{1}(t) \alpha_{2}(t)\right\rangle^{2}$ are positive, Mandel's $Q$-factor in this case is definitely greater than 0 . This ensures 


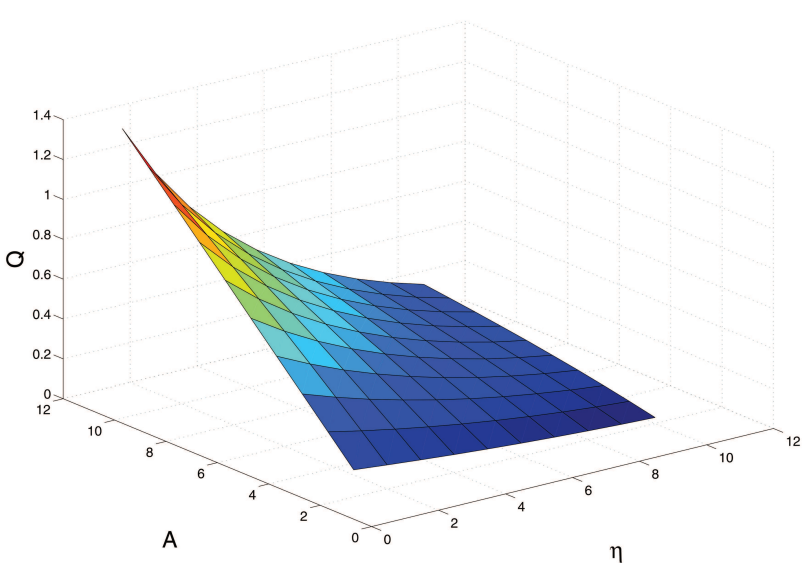

Fig. 7. (Color online) Plot of Mandel's parameter $Q$ versus $\eta$ and the linear gain coefficient $A$ for $\kappa=0.8$

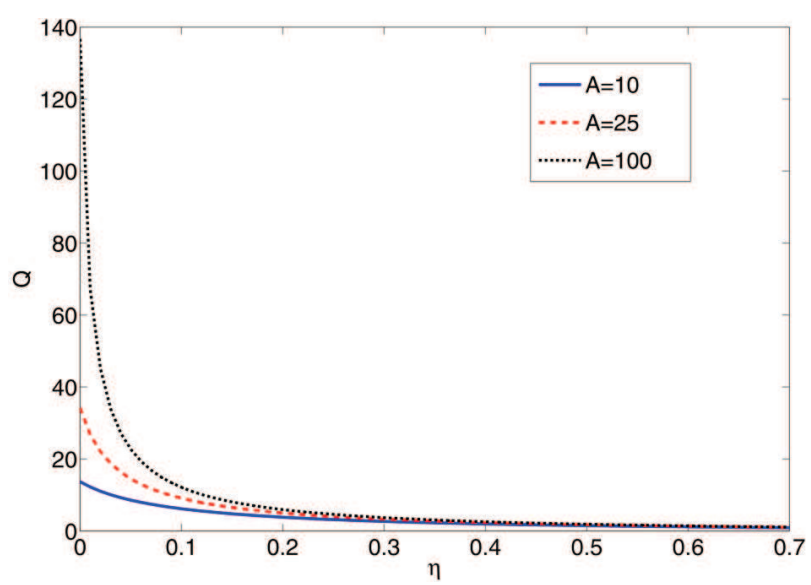

Fig. 8. (Color online) Plots of Mandel's parameter $Q$ versus $\eta$ for $\kappa=0.8$ and the linear gain coefficient $A=10,25$ and 100

that the generated radiation demonstrates the superPoissonian photon statistics while exhibiting the nonclassical properties such as squeezing and entanglement.

Figure 7 shows Mandel's $Q$-factor described by Eq. (47) versus $\eta$ for $A=10$ and $\kappa=0.8$. This figure indicates that Mandel's $Q$-factor for the given system is definitely positive. This implies that the generated laser light demonstrates the super-Poissonian photon statistics. From this plot, we see that Mandel's $Q$ factor increases with the linear gain coefficient at the minimum atomic coherence, $\eta$, which is the maximum squeezing occurring point.

The result presented in Fig. 8 indicates that Mandel's $Q$-factor increases for small values of the atomic coherence $\eta$ and for the cavity damping constant, $\kappa=0.8$ and the linear gain coefficient, $A=10$. The result in this figure indicates that the linear gain coefficient (rate of atomic injection) increases with Mandel's $Q$-factor at the minimum atomic coherence, $\eta$. In these two figures, we observe that the value of Mandel's $Q$-factor is positive.

\subsection{Photon number correlations}

The normalized second-order correlation function for the two-mode light can be expressed as [36]

$g_{\left(n_{1}, n_{2}\right)}^{(2)}(0)=\frac{\left\langle\hat{a}_{1}^{\dagger} \hat{a}_{2}^{\dagger} \hat{a}_{1} \hat{a}_{2}\right\rangle}{\left\langle\hat{a}_{1}^{\dagger} \hat{a}_{1}\right\rangle\left\langle\hat{a}_{2}^{\dagger} \hat{a}_{2}\right\rangle}$.

We realize that the operators in (48) are in the normal order. Therefore, the second-order correlation function can be expressed in terms of the $c$-number variables associated with the normal ordering as

$g_{\left(a_{1}, a\right)_{2}}^{(2)}(0)=1+\frac{\left\langle\alpha_{1}(t) \alpha_{2}(t)\right\rangle^{2}}{\left\langle\alpha_{1}^{*}(t) \alpha_{1}(t)\right\rangle\left\langle\alpha_{2}^{*}(t) \alpha_{2}(t)\right\rangle}$.

We see from Fig. 9 that the correlation of the photon number inccreases with the injected atomic coherence. Moreover, as shown in Fig. 2, the squeezing is maximum in a vicinity of $\eta=0.74$ for $A=10$, where the correlation of the photon number is a little above 2 . We also found that, for $\eta$ very close to 1 , the correlation of the photon number would be significantly larger, since the mean photon number of the light in mode $a_{1}$ is very close to zero, when initially almost all atoms occupy the lower level.

Furthermore, Fig. 10 shows the plots of the linear gain coefficient described by Eq. (49) versus $\eta$ and $A$ for $\kappa=0.8$. It is not difficult to see from this figure that the normalized second-order correlation function increases significantly with the linear gain coefficient and the atomic coherence, $\eta$. However, we have found that the degree of squeezing increases with the linear gain coefficient. Hence, we infer from these results that the correlation between the photon numbers tends to be minimum in regions, where the squeezing is maximum.

\subsection{Intensity difference fluctuations}

The intensity-difference fluctuations allow us to investigate how the difference between the mean photon numbers of the two radiations deviates from each other. This study is based on the assumption that 


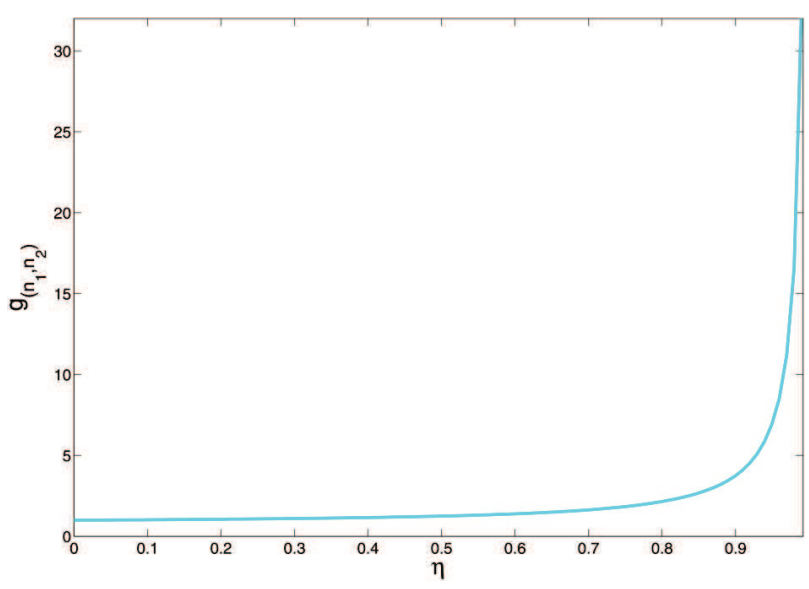

Fig. 9. (Color online) Plots of Mandel's parameter $Q$ versus $\eta$ for $\kappa=0.8$ and the linear gain coefficient $A=10$

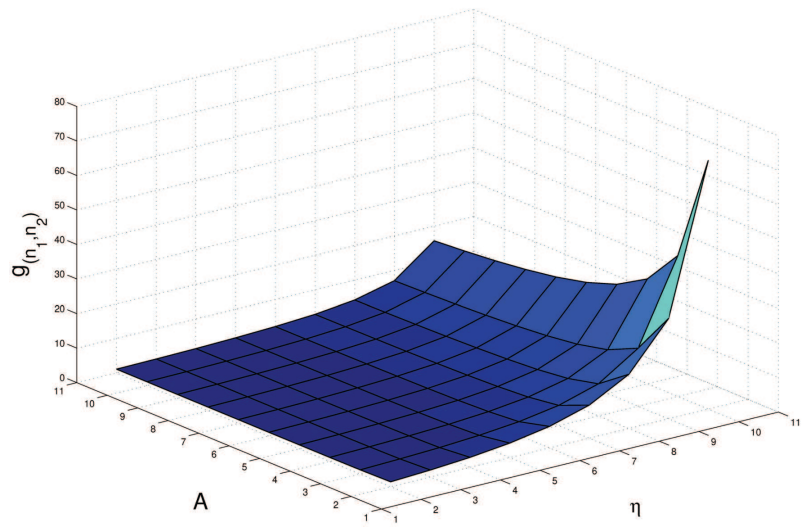

Fig. 10. (Color online) Plot of the photon number correlation function $g_{\left(n_{1}, n_{2}\right)}^{(2)}$ versus $\eta$ and the linear gain coefficient $A$ for $\kappa=0.8$

there is a difference between the mean photon numbers of the two radiations due to the disparity of the absorption-emission mechanism among the involved atomic energy levels. Therefore, the variance of the intensity-difference can be defined as

$\Delta I_{D}^{2}=\left\langle\hat{I}_{D}^{2}\right\rangle-\left\langle\hat{I}_{D}\right\rangle^{2}$,

where the intensity difference is

$\hat{I}_{D}=\hat{a}_{1}^{\dagger} \hat{a}_{1}-\hat{a}_{2}^{\dagger} \hat{a}_{2}$.

Hence, making use of Eq. (51), the $c$-number variance of the intensity-difference takes the form

$\Delta I_{D}^{2}=\left\langle\alpha_{1}^{*}(t) \alpha_{1}(t)\right\rangle\left[1+\left\langle\alpha_{1}^{*}(t) \alpha_{1}(t)\right\rangle\right]+\left\langle\alpha_{2}^{*}(t) \alpha_{2}(t)\right\rangle \times$

$\times\left[1+\left\langle\alpha_{2}^{*}(t) \alpha_{2}(t)\right\rangle\right]-2\left\langle\alpha_{1}(t) \alpha_{2}(t)\right\rangle^{2}$.

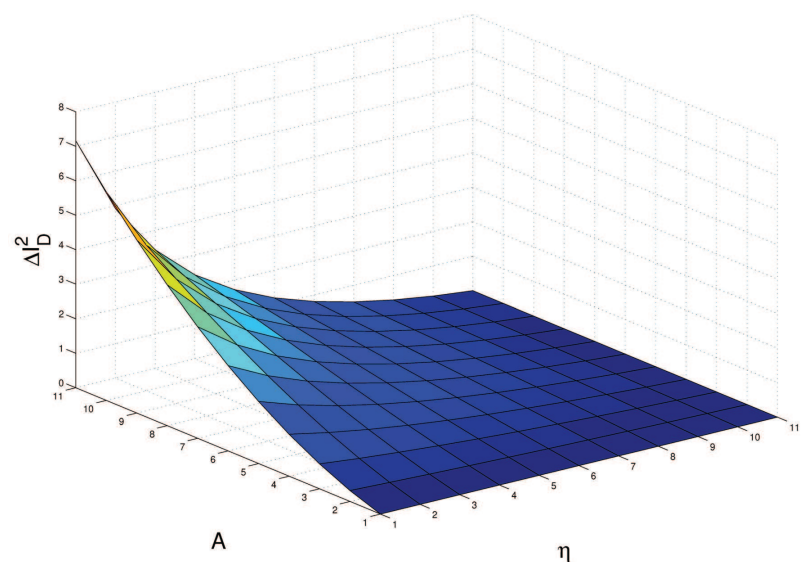

Fig. 11. (Color online) Plots of the variance of the intensitydifference $\Delta I_{D}^{2}$ versus $\eta$ and the linear gain coefficient $A$ for $\kappa=0.8$

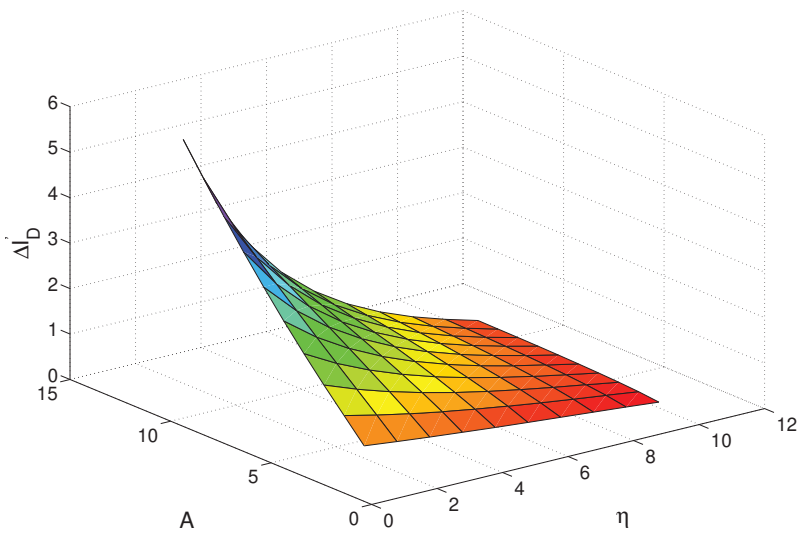

Fig. 12. (Color online) Plots of the variance of the intensitydifference per mean photon number $\Delta I_{D}^{\prime}$ versus $\eta$ for $\kappa=0.8$ and the linear gain coefficient $A=10$

On the other hand, the variance of the intensity difference per mean photon number can be described as $\Delta I_{D}^{\prime}=\sqrt{\frac{\Delta I_{D}^{2}}{\bar{N}}}$.

It is not difficult to see from Fig. 11 that the variance of the intensity difference increases with the linear gain coefficient. But, unlike the correlation between the photon number, it decreases with the injected atomic coherence. In particular, the variance of the intensity difference is found to be zero, when $\eta=1$ for all values of the linear gain coefficient, since there is no possibility for the emission of photons of both modes, when the atoms are initially present on the lower level. In the same way, the variance of the intensity difference turns out to be zero, when 
$A=0$ for all values of $\eta$, since there is no radiation in the cavity. In relation to the previous discussions, one can infer that the variance of the intensity difference would be relatively larger in a region, where the squeezing and entanglement are significant.

Moreover, as shown in Fig. 12, the variance of the intensity difference per mean photon number is found to be less than 1 except for values of $\eta$ and $A$ very close to zero. We observe that $\Delta I^{\prime 2}$ decreases with the injected atomic coherence except near $\eta=1$, where the mean photon number approaches zero.

\section{Conclusions}

In this study, we have analyzed the squeezing, entanglement, and statistical properties of the light produced by a nondegenerate three-level cascade laser coupled to a two-mode vacuum reservoir in the linear and adiabatic approximation schemes in the good cavity limit. Moreover, we apply the master equation describing the dynamics of the optical system. Employing the solutions for the $c$-number cavity mode variables along with the correlation property of noise forces associated with a normal ordering, we obtained the quadrature squeezing, photon entanglement, mean number of photon pairs, Mandels $Q$ factor, second-order correlation functions, and the variance of the intensity difference of the two-mode cavity light.

It is found that the two-mode cavity radiation exhibits squeezing properties under certain conditions pertaining to the injected atomic coherence, where the degree of squeezing increases with the linear gain coefficient. In particular, the squeezing property exists, if the atoms are initially prepared in such a way that there are more atoms on the bottom level, than on the upper level. A relatively better squeezing is found, when a sufficiently large number of atoms is injected into the cavity and when the atoms are initially prepared with a nearly $50-\%$ probability to be on the top level.

We also found that there is a significant entanglement between the states of the light generated in the cavity of a nondegenerate three-level cascade laser due to the strong correlation between the radiation emitted, when the atom decays from the top level to the bottom level via the intermediate level. Generally, we have found that the degree of entanglement for the two-mode cavity light is directly related to the two-mode squeezing. Whenever there is squeez- ing in the two-mode light, there exists the entanglement in the system. The results we have obtained indicate that the entanglement is stronger, when the degree of squeezing is larger. Here, the linear gain coefficient increases with the degree of squeezing. Moreover, we have found that a considerable degree of entanglement of light for very small values of the linear gain coefficient regardless of how atoms are initially prepared.

Furthermore, our calculation of the photon number correlation shows that if the correlation between the states of the emitted light is stronger, the correlation between the photon numbers tends to be smaller. Contrary to this fact, the variance of the intensity difference is found to be relatively larger in a region, where the squeezing and entanglement are significant. We also found that the linear gain coefficient enhance the mean photon number over the laser system. Therefore, the increase in the mean photon number is observed in a region, where the degrees of two-mode squeezing and entanglement are significant making the system under consideration a viable source of intense squeezed, as well as entangled, light.

1. S. Qamar, M. Al-Amri, M.S. Zubairy, Entanglement in a bright light source via Raman-driven coherence. Phys. Rev. A 79, 013831 (2009).

2. J. Anwar, M.S. Zubairy. Quantum-statistical properties of noise in a phase-sensitive linear amplifier. Phys. Rev. A 49, 481 (1994).

3. N.A. Ansari, J.G. Banacloche, M.S. Zubairy. Phasesensitive amplification in a three-level atomic system. Phys. Rev. A 41, 5179 (1990).

4. H. Xiong, M.O. Scully, M.S. Zubairy. Correlated spontaneous emission laser as an entanglement amplifier. Phys. Rev. Lett. 94, 023601 (2005).

5. C.A. Blockley, D.F. Walls. Intensity fluctuations in a frequency down-conversion process with three-level atoms. Phys. Rev. 43, 5049 (1991).

6. N. Lu, F.X. Zhao, J. Bergou. Nonlinear theory of a twophoton correlated-spontaneous-emission laser: A coherently pumped two-level-two-photon laser. Phys. Rev. A 39, 5189 (1989).

7. E. Alebachew. Enhanced squeezing and entanglement in a nondegenerate three-level cascade laser with injected squeezed light. Opt. Commun. 280, 133 (2007).

8. T. Abebe. The quantum analysis of non-degenerate threelevel laser with spontaneous emission and noiseless vacuum reservoir. Ukr. J. Phys. 63, 969 (2018).

9. B. Teklu. Parametric oscillation with the cavity mode driven by coherent light and coupled to a squeezed vacuum reservoir. Opt. Commun. 261, 310 (2006).

ISSN 2071-0194. Ukr. J. Phys. 2021. Vol. 66, No. 7 
10. T. Abebe. Enhancement of squeezing and entanglement in a non-degenerate three-level cascade laser with coherently driven cavity. Ukr. J. Phys. 63, 733 (2018).

11. T. Abebe. Coherently driven nondegenerate three-level laser with noiseless vacuum reservoir. Bulg. J. Phys. 45 357 (2018).

12. T. Abebe, N. Gemechu. Two-level atom with squeezed light from optical parametric oscillators. Ukr. J. Phys. 63, 600 (2018).

13. Ch. Gashu, T. Abebe. Externally induced entanglement amplification in a coherently pumped emission of laser with parametric amplifier and coupled to squeezed vacuum reservoir. Phys. Scr. 95, 075105 (2020).

14. T. Abebe, N. Gemechu, Ch. Gashu, K. Shogile, S. Hailemariam, Sh. Adisu. The quantum analysis of nonlinear optical parametric processes with thermal reservoirs. Int. J. Opt. 2020, 7198091 (2020).

15. T. Abebe, N. Gemechu, K. Shogile, S. Hailemariam, Ch. Gashu, Sh. Adisu. Entanglement quantification using various inseparability criteria for correlated photons. Rom. J. Phys. 65, 107 (2020).

16. A. Einstein, B. Podolsky, R. Rosen. Can quantum mechanical description of physical reality be considered complete? Phys. Rev. 47, 777 (1935).

17. J.S. Bell. On the Einstein-Podolsky-Rosen paradox. Physics 1, 195 (1964).

18. J.M. Liu, B.S. Shi, X.F. Fan, J. Li, G.C. Guo. Wigner function description of continuous variable entanglement swapping. J. Opt. B: Quant. Semiclass. Opt. 3, 189 (2001).

19. S.L. Braunstein, H.J. Kimble. Dense coding for continuous variables. Phys. Rev. A 61. 042302 (2000).

20. S. Lloyd, S.L. Braunstein. Quantum computation over continuous variables. Phys. Rev. Lett. 82, 1784 (1999).

21. S.L. Braunstein. Quantum error correction for communication with linear optics. Nature 394, 47 (1998).

22. T.C. Ralph. Continuous variable quantum cryptography. Phys.Rev. A 61, 010302 (2000).

23. T. Jennewein, C. Simon, G. Weihs, H. Wein-furter, A. Zeilinger. Quantum cryptography with entangled photons. Phys. Rev. Lett. 84, 4729 (2000).

24. C.H. Bennett, D.P. DiVincenzo. Quantum information and computation. Nature 404, 247 (2000).

25. S. Barzanjeh, S. Pirandola, C. Weedbrook. Continuousvariable dense coding by optomechanical cavities. Phys. Rev. A 88, 042331 (2013).

26. N. Ganguly, S. Adhikari, A.S. Majumdar, J. Chatterjee. Entanglement witness operator for quantum teleportation. Phys. Rev. Lett. 107, 270501 (2011).

27. C. Branciard, N. Brunner, H. Buhrman, R. Cleve, N. Gisin, S. Portmann, D. Rosset, M. Szegedy. Classical simulation of entanglement swapping with bounded communication. Phys. Rev. Lett. 109, 100401 (2012).

28. T. Kitagawa, A. Aspect, M. Greiner, E. Demler. Phasesensitive measurements of order parameters for ultra- cold atoms through two-particle interferometry. Phys. Rev. Lett. 106, 115302 (2011).

29. S. Koike, H. Takahashi, H. Yonezawa, N. Takei, S.L. Braunstein, T. Aoki, A. Furusawa. Phys. Rev. Lett. 96, 060504 (2006).

30. R.T. Thew, W.J. Munro. Entanglement manipulation and concentration. Phys. Rev. A 63, 030302(R)(2001).

31. T. Kishore, P. Anirban, S. Biswajit, J. Peřina. Higher-order nonclassicalities in a codirectional nonlinear optical coupler: Quantum entanglement, squeezing, and antibunching. Phys. Rev. A 90, 013808 (2014).

32. N. Javid, T. Kishore, P. Anirban, S. Banerjee. Probing nonclassicality in an optically driven cavity with two atomic ensembles. Phys. Rev. A 97, 063840 (2018).

33. L.M. Duan, G. Giedke, J.I. Cirac, P. Zoller. Inseparability criterion for continuous variable systems. Phys. Rev. Lett. 84, 2722 (2000).

34. Y.H. Ma, Q.X. Mu, G.H. Yang, L. Zhou. Enhanced continuous-variable entanglement by a self-phase-locked type-II optical parameter oscillator with feedback loops. Phys. B: At. Mol. Opt. Phys. 41, 215502 (2008).

35. G. Vidal, R.F. Wener. Computable measure of entanglement. Phys. Rev. A 65, 032314 (2002).

36. M. Fox. Quantum Optics: An Introduction (Oxford University Press, 2006).

37. Ch. Gashu, E. Mosisa, T. Abebe. Entanglement quantification of correlated photons generated by three-level laser with parametric amplifier and coupled to a two-mode vacuum reservoir. Adv. Math. Phys. 2020, 9027480 (2020).

38. G. Adesso, A. Serafini, F. Illuminati. Extremal entanglement and mixedness in continuous variable systems. Phys. Rev. A 70, 022318 (2004).

Received 27.05.20

\section{Т. Абебе, К. Гашу}

\section{ГЕНЕРАЦІЯ ЗАПЛУТАНОГО}

СВІТЛА НЕВИРОДЖЕНИМ ТРИРІВНЕВИМ

ЛАЗЕРОМ, З'ЄДНАНИМ 3 ДВОМОДОВИМ

\section{ВАКУУМНИМ РЕЗЕРВУАРОМ}

Вивчаються квантові властивості невиродженого трирівневого каскадного лазера, з'єднаного з двомодовим вакуумним резервуаром із застосуванням відповідного основного рівняння і стохастичних диференційних рівнянь, асоційованих $з$ нормальним упорядкуванням. Зокрема, досліджено посилення стиснення i заплутаності фотонів двомодового резервуара. Показано, що обидві моди сильно заплутані, i ступінь заплутаності безпосередньо пов'язаний з двомодовим стисненням. Більш того, стиснення і заплутаність зростають зі збільшенням швидкості атомного накачування.

Kлюч о в $i$ слов в: атомна когерентність, квадратурне стиснення, заплутаність, середне число фотонів. 